\title{
$\mathrm{Cu} @ P t / M W C N T s-M_{2} O_{2}$ 电催化剂的制备及电催化性能研究
}

\author{
于书平娄群刘润婷韩克飞汪中明朱红 \\ (北京化工大学化工资源有效利用国家重点实验室 北京 100029)
}

\begin{abstract}
摘要 通过浸渍还原法, 以乙二醇作为还原剂, 以 $\mathrm{H}_{2} \mathrm{PtCl}_{6} \cdot 6 \mathrm{H}_{2} \mathrm{O}$ 作为 $\mathrm{Pt}$ 的前驱体制备了 $\mathrm{Cu} @ \mathrm{Pt} / \mathrm{MWCNTs}$ 核壳型电催 化剂; 通过水热法, 以 $\mathrm{KMnO}_{4}$ 和 $\mathrm{Mn}\left(\mathrm{NO}_{3}\right)_{2}$ 作为锰源制备了 $\alpha-\mathrm{MnO}_{2}$ 和 $\beta-\mathrm{MnO}_{2}$, 并把 $\mathrm{Cu} @ \mathrm{Pt} / \mathrm{MWCNTs}$ 核壳型电催化 剂与二氧化锰进行掺杂制得 $\mathrm{Cu} @ \mathrm{Pt} / \mathrm{MWCNTs}-\mathrm{MnO}_{2}$ 复合材料. 利用 XRD, SEM, TEM 对复合材料的结构和形貌进行表 征, 利用循环伏安测试曲线和阴极极化曲线等电化学测试方法对电催化剂的性能进行测试. 结果表明, 电催化剂中 $\mathrm{Cu} @ \mathrm{Pt}$ 纳米颗粒为核壳型, 粒径为 $6 \sim 8 \mathrm{~nm}, \mathrm{MnO}_{2}$ 的晶型为 $\alpha-\mathrm{MnO}_{2}$ 和 $\beta-\mathrm{MnO}_{2}$; 另外, $\mathrm{Cu} @ \mathrm{Pt} / \mathrm{MWCNTs}-\mathrm{MnO}_{2}$ 复合材 料具有良好的催化性能, 其中 $\mathrm{Cu} @ \mathrm{Pt} / \mathrm{MWCNTs}-\beta-\mathrm{MnO}_{2}$ 电催化剂的电化学性能较好, 具有较大的电化学活性面积, 为 $71.1 \mathrm{~m}^{2} \cdot \mathrm{g}^{-1}$, 同时对 $\mathrm{MnO}_{2}$ 促进氧还原的机理进行了初步探讨.

关键词 质子交换膜燃料电池; 核壳型; 二氧化锰; 电催化剂; 电化学活性面积
\end{abstract}

\section{Synthesis and Electrocatalytic Performance of $\mathrm{Cu} @ \mathrm{Pt} / \mathrm{MWCNTs}-\mathrm{MnO}_{2}$ Electrocatalyst}

\author{
Yu, Shuping Lou, Qun Liu, Runting Han, Kefei Wang, Zhongming Z Zhu, Hong* \\ (State Key Laboratory of Chemical Resource Engineering, Beijing University of Chemical Technology, Beijing 100029)
}

\begin{abstract}
The core-shell structure catalysts with reduced Pt loading and improved catalytic activity which are inter-metallic Pt electrocatalyst with a cheap transition metal core have become a critical issue in fuel cells. In this study, Cu@Pt/MWCNTs core-shell electrocatalyst was prepared through the impregnation chemical reduction method, by using ethylene glycol as the reducing agent, $\mathrm{H}_{2} \mathrm{PtCl}_{6}$ as a precursor of $\mathrm{Pt}$ and $\mathrm{CuSO}_{4}$ as a precursor of $\mathrm{Cu}$. At the appropriate temperature and $\mathrm{pH}$ (adjusted by $\mathrm{KOH} / \mathrm{EG}$ solution), $\mathrm{Cu}$ nanoparticles were reduced on the surface of MWCNTs $\left(140{ }^{\circ} \mathrm{C}, \mathrm{pH}=10\right)$, and then Pt atoms were deposited on the surface of $\mathrm{Cu}$ nanoparticles $\left(90{ }^{\circ} \mathrm{C}, \mathrm{pH}=7 \sim 8\right) . \alpha-\mathrm{MnO}_{2}$ and $\beta-\mathrm{MnO}_{2}$ were prepared by hydrothermal method with $\mathrm{KMnO}_{4}$ and $\mathrm{Mn}\left(\mathrm{NO}_{3}\right)_{2}$ as the manganese source, the reaction time of $\alpha-\mathrm{MnO}_{2}$ and $\beta-\mathrm{MnO}_{2}$ is $8 \mathrm{~h}$ and $72 \mathrm{~h}$, respectively. The obtained manganese dioxide was doped onto $\mathrm{Cu} @ \mathrm{Pt} / \mathrm{MWCNT}$ under ultrasound to make $\mathrm{Cu} @ \mathrm{Pt} /$ MWCNTs- $\mathrm{MnO}_{2}$ composite catalysts. The structure and morphology of $\mathrm{Cu} @ \mathrm{Pt} / \mathrm{MWCNTs}$ and $\mathrm{MnO}_{2}$ were characterized by $\mathrm{XRD}$, SEM and TEM, electrochemical performances were investigated by cyclic voltammetry, cathodic polarization and other electrochemical methods. In order to discuss the oxygen reduction method of $\mathrm{Cu} @ \mathrm{Pt} / \mathrm{MWCNTs}-\mathrm{MnO}_{2}, \mathrm{RRDE}$ (rotating ring-disk electrode) was further used to examine the catalytic oxygen reduction reactions of $\mathrm{Cu} @ \mathrm{Pt} / \mathrm{MWCNTs}-\mathrm{MnO}_{2}$. Our results demonstrated that the $\mathrm{Cu} @$ Pt nanoparticles have the core-shell structure. The diameter of the nanoparticles is about $6 \sim 8 \mathrm{~nm}$. The crystal morphology of $\mathrm{MnO}_{2}$ is $\alpha-\mathrm{MnO}_{2}$ and $\beta-\mathrm{MnO}_{2}$. It was also found that the $\mathrm{Cu} @ \mathrm{Pt} /$ MWCNTs- $\beta-\mathrm{MnO}_{2}$ composite catalysts have better catalytic performance and higher electrochemical activity surface (up to $\left.71.1 \mathrm{~m}^{2} \cdot \mathrm{g}^{-1}\right)$. The RRDE results for the ORR indicate that, the main reaction of $\mathrm{Cu} @ \mathrm{Pt} / \mathrm{MWCNTs}-\beta-\mathrm{MnO}_{2}$ catalyst is the direct reduction of $\mathrm{O}_{2}$ to $\mathrm{H}_{2} \mathrm{O}$ which is same as the mechanism of $\mathrm{Cu} @$ Pt/MWCNTs catalyst, both with a four-electron charge transfer. Moreover, the mechanism of $\mathrm{MnO}_{2}$-promoting effects for the oxygen reduction was also discussed in this paper.
\end{abstract}

Keywords PEMFC core-shell; manganese dioxide; electrocatalyst; oxygen reduction; electrochemical active surface

\section{1 引言}

随着全世界范围内对清洁能源需求的日益增加, 电 催化剂作为质子交换膜燃料电池(PEMFC)的核心组分 被不断广泛而深入的研究 ${ }^{[1 \sim 5]}$. 核壳型电催化剂作为一 种微观有序的催化剂结构已经得到了许多学者的广泛
关注，形成核壳结构的过渡金属与贵金属能够形成协同 作用以提高催化性能和提高阴极氧还原电位的同时，还 能很大程度上地减少 PEMFC 电催化剂中贵金属的用 量 $^{[6 \sim 9]}$. Yang 等 ${ }^{[10]}$ 合成碳载 $\mathrm{Co} @ \mathrm{Pt}$ 纳米颗粒催化剂展示 了良好的催化水解 $\mathrm{NH}_{3} \mathrm{BH}_{3}$ 的性质. 高分辨透射电镜及

*E-mail: zhuho128@126.com; yusp@mail.buct.edu.cn

Received October 16, 2012; published October 31, 2012.

Supporting information for this article is available free of charge via the Internet at http://sioc-journal.cn.

Project supported by the National Natural Science Foundation of China (No. 21176022), the International S\&T Cooperation Program of China (No. 2009DFA63120), and the National Defense Basic Scientific Research Program of China (No. A1420110023).

项目受国家自然科学基金(No. 21176022)、国际合作(No. 2009DFA63120)和国防科学研究(No. A1420110023)资助. 
分析展现了明显的核壳型的结构, 通过循环伏安测试表 明, Co@Pt 纳米颗粒的催化剂比纯 Pt 电催化剂具有更好 的催化活性. Wang 等 ${ }^{[11]}$ 制备了无碳载体 $\mathrm{Ni@Pt}$ 核壳型 电催化剂, 其中 $\mathrm{Pt}$ 的载量为 $16.7 \mathrm{wt} \%$, 并且其催化氧化 还原的能力高于商业 BASF 公司的 $20 \mathrm{wt} \%$ 的铂碳催化 剂. $\mathrm{Zhu}$ 等 ${ }^{[12]}$ 用两步化学还原法合成了 $\mathrm{XC}-72 \mathrm{R}$ 为载体 的 $\mathrm{Cu} @ \mathrm{Pt} / \mathrm{C}$ 催化剂, 该催化剂 Pt 的电化学活性面积达 到 $53.81 \mathrm{~m}^{2} \cdot \mathrm{g}^{-1}$, 同时解决了引起电池效率下降和输出 电压增大的高电位下氧还原极化问题. 二氧化锰在电化 学电池材料和催化剂中具有广泛的应用, 二氧化锰具有 多种的晶型结构, 主要报道的有隐钾锰矿 $\left(\alpha-\mathrm{MnO}_{2}\right)$ 、软 锰矿 $\left(\beta-\mathrm{MnO}_{2}\right)$ 等晶型, 它们具有不同的电化学性质 ${ }^{[13 ~ 15] . ~}$ 近年来研究发现, 二氧化锰表面具有富集氧气的能力, 对于燃料电池阴极催化剂催化氧还原具有良好的应用 前景 ${ }^{[16,17]}$. $\mathrm{Yu}$ 等 ${ }^{[18]}$ 利用一种简单的方法合成了茧状、海 胆状和巢状的 $\mathrm{Pt} / \mathrm{MnO}_{2}$ 电催化剂用于氧化分解甲醛, $\mathrm{Pt}$ 的粒径约为 $1 \sim 4 \mathrm{~nm}$, 当 $\mathrm{Pt}$ 的含量为 $2 \%$ 的时候催化剂 具有最佳的甲醛氧化能力, 并讨论了 $\mathrm{Pt}$ 与二氧化锰的 协作催化效应. Trogadas 等 ${ }^{[19]}$ 利用湿化学法制备了 $\mathrm{Pt} / \mathrm{MnO}_{2} / \mathrm{C}$, 对催化反应过程中对分解过氧化氢的量的 检测，结果表明当二氧化锰的含量为 $5 \%$ 的时候，能够 达到分解 $50 \%$ 60\%过氧化氢的作用, 达到保护质子交 换膜的作用. Zhou 等 ${ }^{[17]}$ 通过在碳纳米管的表面负载水 合二氧化锰和 $\mathrm{Pt}$ (PtRu 合金)合成了 $\mathrm{Pt} / \mathrm{MnO}_{2} / \mathrm{CNT}$ 和 $\mathrm{PtRu} / \mathrm{MnO}_{2} / \mathrm{CNT}$ 电催化剂, $\mathrm{Pt} / \mathrm{MnO}_{2} / \mathrm{CNT}$ 比 $\mathrm{Pt} / \mathrm{CNT}$ 显 示了较高的电化学活性面积和较好的甲醇电氧化活性, $\mathrm{PtRu} / \mathrm{MnO}_{2} / \mathrm{CNT}$ 不仅显示了对甲醇良好的电氧化活性 和 $901 \mathrm{~A} / \mathrm{g}_{\mathrm{Pt}}$ 阳极峰电流, 而且展现了较低 $\mathrm{CO}$ 氧化起始 电位 $(0.33 \mathrm{~V}$ vs. $\mathrm{Ag} / \mathrm{AgCl})$ 和峰电位 $(0.49 \mathrm{~V}$ vs. $\mathrm{Ag} / \mathrm{AgCl})$ 等良好的 $\mathrm{CO}$ 氧化能力.

本文通过两步化学还原法合成了 $\mathrm{Cu} @ \mathrm{Pt}$ 纳米颗粒 并负载在多壁碳纳米管(MWCNTs)上，同时通过控制水 热反应的时间制备了 $\alpha-\mathrm{MnO}_{2}$ 和 $\beta-\mathrm{MnO}_{2}$ 两种晶型的二 氧化锰, 将这两种晶型的 $\mathrm{MnO}_{2}$ 与 $\mathrm{Cu} @ \mathrm{Pt} / \mathrm{MWCNTs}$ 进 行掺杂, 对掺杂物的电化学性能, 催化性能进行了测试, 探讨了其催化机理.

\section{2 结果与讨论}

\section{$2.1 X R D$ 测试}

图 1 为所制备的电催化剂的 XRD 谱图. 对比图 1(a) 和 1(b), Cu@Pt/MWCNTs 电催化剂比 Pt/MWCNTs 电催 化剂的特征衍射峰的 $2 \theta$ 角有一定程度的正移, 由于 $\mathrm{Pt}$ 的晶胞里面的原子被原子半径比其小的 $\mathrm{Cu}$ 原子取代, 造成了铂的晶胞收缩和晶胞体积减小, 即晶格收缩, 晶 格常数变小 ${ }^{[20]}$, 根据布拉格方程, 当晶面间距 $d$ 变小之 后, 导致 $\theta$ 角增大. 因此可以初步确定 $\mathrm{CuPt}$ 核壳或合金 体系已经形成. 根据 Scherrer 公式 ${ }^{[21]}$ 计算可知, $\mathrm{Cu} @ \mathrm{Pt}$ / MWCNTs 电催化剂纳米颗粒的平均粒径为 $8 \sim 9 \mathrm{~nm}$. 图 2(a)和 2(b) 分别为 $\alpha-\mathrm{MnO}_{2}$ 和 $\beta-\mathrm{MnO}_{2}$ 的 XRD 谱图，通过 比较可以看到两种晶型的二氧化锰特征衍射峰明显, 无 杂峰, 产物纯净, 且 $\beta-\mathrm{MnO}_{2}$ 具有更加尖锐的峰型, 说明 $\beta-\mathrm{MnO}_{2}$ 结晶度更高, 结晶颗粒尺寸较大.

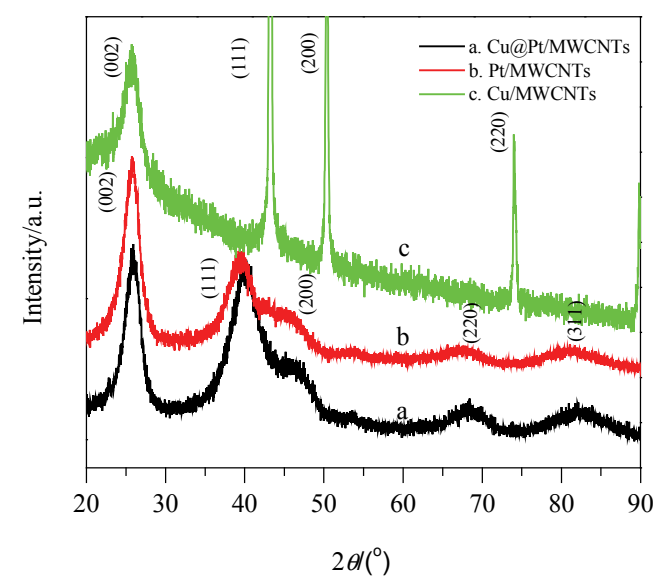

图 1 电催化剂的 XRD 谱图

Figure 1 X-ray diffraction patterns of electrocatalysts (a) $\mathrm{Cu} @ \mathrm{Pt} / \mathrm{MWCNTs}$; (b) Pt/MWCNTs; (c) Cu/MWCNTs
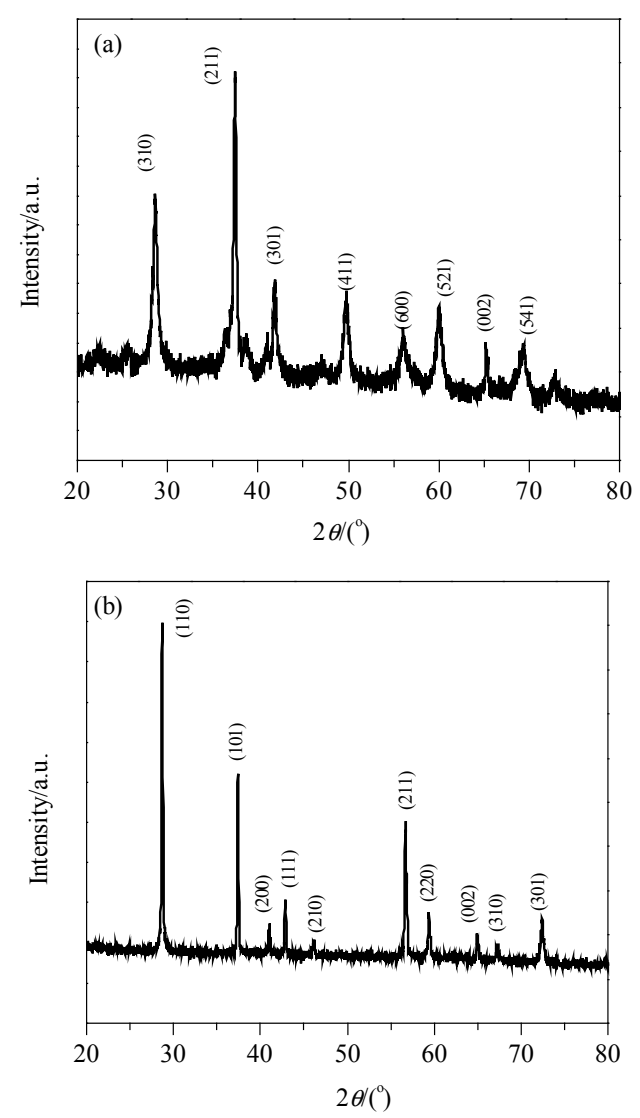

图 2 不同晶型二氧化锰的 XRD 谱图

Figure 2 XRD patterns of different $\mathrm{MnO}_{2}$ crystal structure (a) $\alpha-\mathrm{MnO}_{2} ;$ (b) $\beta-\mathrm{MnO}_{2}$

\subsection{TEM 和 SEM 测试}

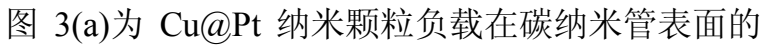


透射电镜图, 可以看到, $\mathrm{Cu} @ \mathrm{Pt}$ 颗粒在碳纳米管的表面 相对均一, 分散性良好. 图 3(b)为 $\mathrm{Cu} @ \mathrm{Pt}$ 纳米颗粒的高 分辨透射电镜图, 该颗粒形状为圆形或椭圆形, 颗粒大 小约为 $6 \sim 8 \mathrm{~nm}$, 纳米颗粒边缘的晶格间距为 $0.228 \mathrm{~nm}$, 纳米颗粒中心的晶格间距约为 $0.222 \mathrm{~nm}$, 介于 $\operatorname{Pt}(111)$ 晶面晶格间距(0.228 nm) 与 $\mathrm{Cu}(111)$ 晶面晶格间距(0.219 $\mathrm{nm})$ 之间, 说明 $\mathrm{Cu} @ \mathrm{Pt}$ 纳米颗粒形成了以铜为核, 铂为 壳的核壳型结构. $\alpha-\mathrm{MnO}_{2}$ 和 $\beta-\mathrm{MnO}_{2}$ 的 TEM 和 SEM 图 如图 4 所示. 由图 4(a,b)得知 $\alpha-\mathrm{MnO}_{2}$ 呈线性, 直径约为 $20 \sim 40 \mathrm{~nm}$, 长度约为 $2 \sim 4 \mu \mathrm{m}$, 图 4(c, d) 中的 $\beta-\mathrm{MnO}_{2}$ 呈六棱柱的形状, 直径约为 $1.5 \mu \mathrm{m}$ 左右, 长度大约为 $4 \sim 6 \mu \mathrm{m}$. 通过延长水热反应时间, $\alpha-\mathrm{MnO}_{2}$ 的晶体可以 转化为 $\beta-\mathrm{MnO}_{2}$ 晶体.
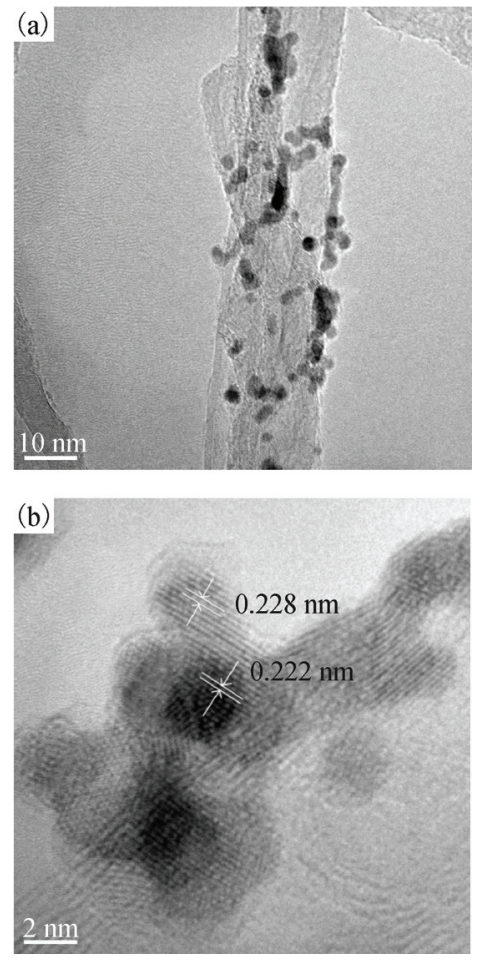

图 $3 \mathrm{Cu} @ \mathrm{Pt} / \mathrm{MWCNTs}$ 电催化剂高分辨透射电镜图

Figure 3 HRTEM image of $\mathrm{Cu} @$ Pt electrocatalyst (a) $\mathrm{Cu} @ \mathrm{Pt} / \mathrm{MWCNTs}$; (b) $\mathrm{Cu} @$ Pt nanoparticles

\section{3 电化学测试}

将掺杂有 $\alpha-\mathrm{MnO}_{2}$ 和 $\beta-\mathrm{MnO}_{2}$ 的 $\mathrm{Cu} @ \mathrm{Pt} / \mathrm{MWCNTs}$ 电催化剂与 $\mathrm{Cu} @ \mathrm{Pt} / \mathrm{MWCNTs}$ 电催化剂分别进行循环伏 安扫描测试(图 5), 可以看出, 掺杂有 $\mathrm{MnO}_{2}$ 的 $\mathrm{Cu} @ \mathrm{Pt}$ / MWCNTs 电催化剂均具有较高的电催化活性, 且掺杂 $\beta-\mathrm{MnO}_{2}$ 的电催化剂的催化活性更好一些. 根据下面的 公式可计算出 Pt 催化剂的电化学活性表面积 ${ }^{[22]}$.

$$
\operatorname{EAS}\left(\mathrm{m}^{2} \cdot \mathrm{g}^{-1}\right)=Q_{\mathrm{H}} \cdot(2.1 \times[\mathrm{Pt}])^{-1}
$$

式中 $[\mathrm{Pt}]$ 为玻碳电极表面 $\mathrm{Pt}$ 的含量, 单位为 $\mathrm{g} \bullet \mathrm{m}^{-2}$, $Q_{\mathrm{H}}\left(\mathrm{C} \cdot \mathrm{m}^{-2}\right)$ 是每平方米铂表面氢的吸附和脱附平均电量. 各催化剂的电化学活性面积如表 1 , 其中 $\mathrm{Cu} @ \mathrm{Pt}$ / MWCNTs- $\beta-\mathrm{MnO}_{2}$ 电催化剂的电化学活性面积最大为
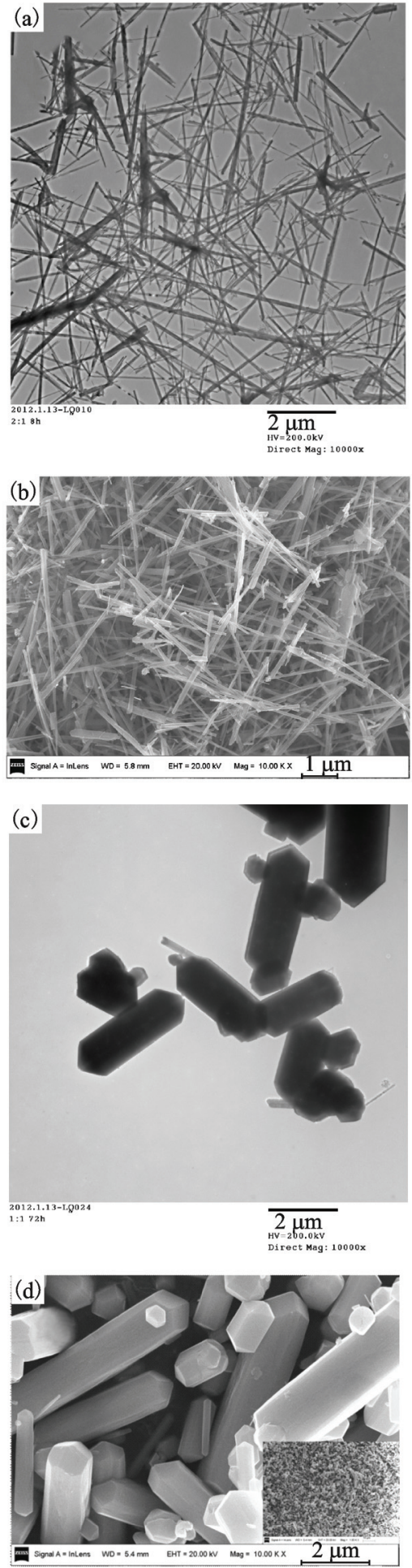

图 4 不同晶型二氧化锰的 TEM 和 SEM 图

Figure 4 TEM and SEM images of different $\mathrm{MnO}_{2}$ crystal structure (a) TEM image of $\alpha-\mathrm{MnO}_{2}$; (b) SEM image of $\alpha-\mathrm{MnO}_{2}$; (c) TEM image of $\beta-\mathrm{MnO}_{2} ;$ (d) SEM image of $\beta-\mathrm{MnO}_{2}$

$71.1 \mathrm{~m}^{2} \cdot \mathrm{g}^{-1} \cdot \mathrm{MnO}_{2}$ 掺杂能够提高催化剂的电化学活性面 积是由于：(1)二氧化锰中含有少量的结合水，这些结合 水为电子和质子的传递提供了无障碍和快速的通道, 有 利于 $\mathrm{Cu} @ \mathrm{Pt} / \mathrm{MWCNTs}-\mathrm{MnO}_{2}$ 电催化剂表面氢的吸附和 脱附, 即提高了电化学活性面积; (2) $\mathrm{Cu} @ \mathrm{Pt}$ 纳米颗粒与 
二氧化锰具有协同作用，一方面，二氧化锰较大的表面 能够吸附更多的氢质子和氧分子以提高氢质子和氧分 子与 $\mathrm{Cu} @ \mathrm{Pt}$ 纳米颗粒接触的机会 ${ }^{[19,23]}$, 另一方面, $\mathrm{Cu} @ \mathrm{Pt}$ 纳米颗粒能够对二氧化锰的氧化还原反应起到 重要作用, $\mathrm{Cu} @ \mathrm{Pt}$ 纳米颗粒可修饰二氧化锰体相与表面 的空穴交换率, 促进氧空位从体相到氧化物表面的迁 移, 促进氧气的吸附, 以达到提高二氧化锰的反应活性 的作用 ${ }^{[19]}$.

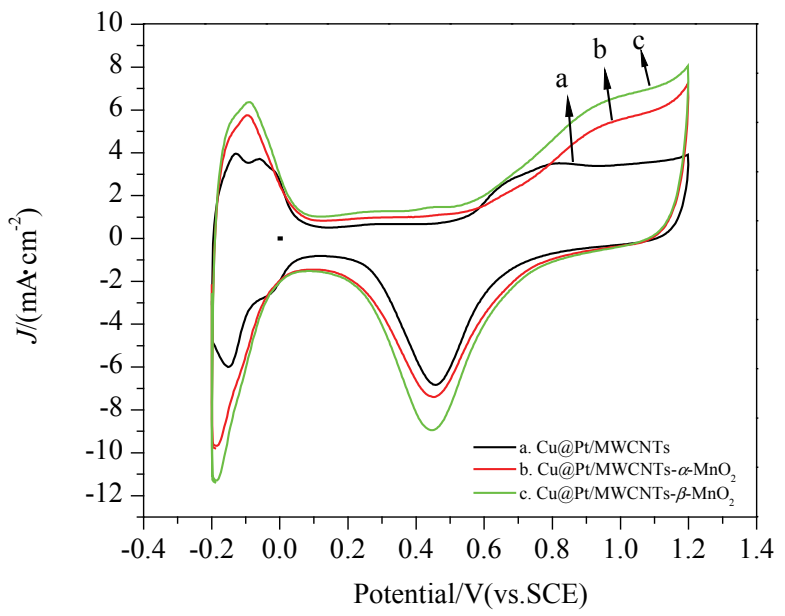

图 5 不同电催化剂的循环伏安扫描曲线

Figure 5 Cyclic voltammograms of different electrocatalysts (a) $\mathrm{Cu} @$ Pt/MWCNTs; (b) $\mathrm{Cu} @ \mathrm{Pt} / \mathrm{MWCNTs}-\alpha-\mathrm{MnO}_{2} ;$ (c) $\mathrm{Cu} @ \mathrm{Pt} /$ MWCNTs- $\beta-\mathrm{MnO}_{2}$. Electrolyte: $0.5 \mathrm{~mol} \cdot \mathrm{L}^{-1} \mathrm{H}_{2} \mathrm{SO}_{4}$. Scanning speed: 50 $\mathrm{mV} \cdot \mathrm{s}^{-1}$

表 1 不同电催化剂的电化学活性面积(EAS)

Table 1 Electrochemical active surface (EAS) of different electrocatalysts

\begin{tabular}{cccc}
\hline $\begin{array}{c}\mathrm{Cu} @ \mathrm{Pt} / \\
\mathrm{MWCNTs}\end{array}$ & $\begin{array}{c}\mathrm{Cu} @ \mathrm{Pt} / \\
\mathrm{MWCNTs}-\alpha-\mathrm{MnO}_{2}\end{array}$ & $\begin{array}{c}\mathrm{Cu} @ \mathrm{Pt} / \\
\mathrm{MWCNTs}-\beta-\mathrm{MnO}_{2}\end{array}$ \\
\hline $\begin{array}{c}\mathrm{EAS} / \\
\left(\mathrm{m}^{2} \cdot \mathrm{g}^{-1}\right)\end{array}$ & 48.6 & 65.2 & 71.1 \\
\hline
\end{tabular}

图 6 为电催化剂在外加电压为 $0.5 \mathrm{~V}$ 时的计时电流 图, 可以看出 $\mathrm{Cu} @ \mathrm{Pt} / \mathrm{MWCNTs}-\beta-\mathrm{MnO}_{2}$ 电催化剂的稳 态电流均大于相应的 $\mathrm{Cu} @ \mathrm{Pt} / \mathrm{MWCNTs}$ 和 Pt/MWCNTs 电 催化剂, 说明金属核壳型结构的形成以及 $\beta-\mathrm{MnO}_{2}$ 的掺 杂有效地提高电催化剂的催化活性, 与循环伏安测试结 果一致.

图 7 为电催化剂的阴极极化曲线图. 掺杂有 $\alpha-\mathrm{MnO}_{2}$ 和 $\beta-\mathrm{MnO}_{2}$ 的 $\mathrm{Cu} @ \mathrm{Pt} / \mathrm{MWCNTs}$ 比 $\mathrm{Cu} @ \mathrm{Pt} / \mathrm{MWCNTs}$ 氧的 起始还原电位分别正移了 9 和 $25 \mathrm{mV}$, 说明 $\mathrm{MnO}_{2}$ 的掺 杂能够促进氧气的还原, 使电催化剂在电化学反应过程 中产生更小的过电位. 掺杂 $\beta-\mathrm{MnO}_{2}$ 比掺杂 $\alpha-\mathrm{MnO}_{2}$ 的电 催化剂具有更强的氧还原能力, 是由于 $\beta-\mathrm{MnO}_{2}$ 比 $\alpha-\mathrm{MnO}_{2}$ 晶体具有更大的表面积, 晶体表面具有更强的 富集 $\mathrm{Mn}^{3+}$ 的能力 ${ }^{[16]}$, 促进氧的还原. 从它们的交流阻 抗测试谱图(图 8)分析得知, 掺杂 $\beta-\mathrm{MnO}_{2}$ 的电催化剂在

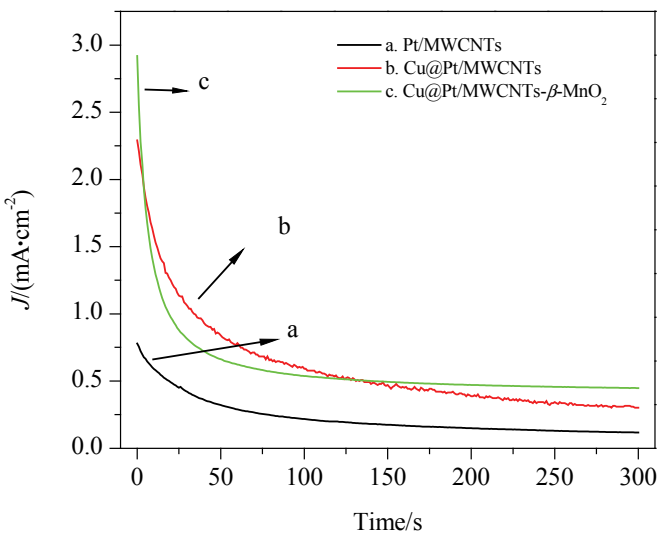

图 6 不同电催化剂的计时电流曲线

Figure 6 Chronoamperometry curve of different electrocatalysts (a) $\mathrm{Pt} / \mathrm{MWCNTs} ; \quad$ (b) $\mathrm{Cu} @ \mathrm{Pt} / \mathrm{MWCNTs} ; \quad$ (c) $\mathrm{Cu} @ \mathrm{Pt} / \mathrm{MWCNTs}-\beta-\mathrm{MnO}_{2}$ Electrolyte: $0.5 \mathrm{~mol} \cdot \mathrm{L}^{-1} \mathrm{H}_{2} \mathrm{SO}_{4}$. Scanning speed: $50 \mathrm{mV} \cdot \mathrm{s}^{-1}$

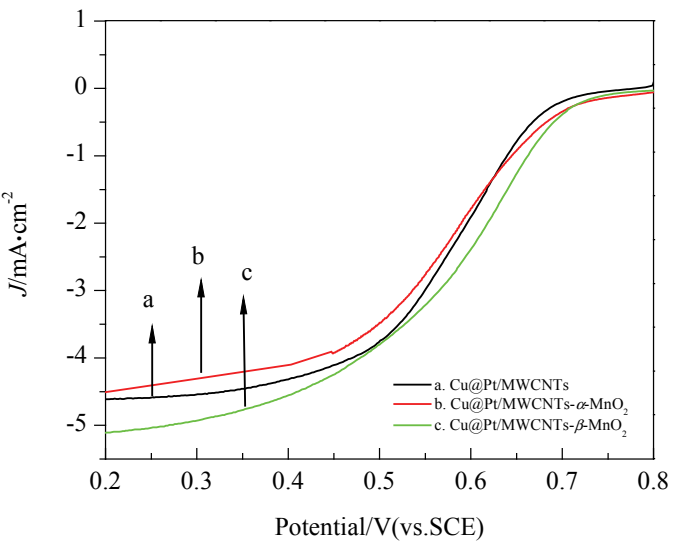

图 7 不同电催化剂的阴极极化曲线

Figure 7 Polarization curves of different electrocatalysts $\mathrm{Cu} @$ Pt/MWCNTs; (b) $\mathrm{Cu} @ \mathrm{Pt} / \mathrm{MWCNTs}-\alpha-\mathrm{MnO}_{2}$; (c) $\mathrm{Cu} @ \mathrm{Pt} / \mathrm{MWCNTs-}$ $\beta$ - $\mathrm{MnO}_{2}$. Electrolyte: $\mathrm{O}_{2}$-saturated $0.5 \mathrm{~mol} \cdot \mathrm{L}^{-1} \mathrm{H}_{2} \mathrm{SO}_{4}$. Sweep rate: $2 \mathrm{mV} \cdot \mathrm{s}^{-1}$. Rotation rate: $1600 \mathrm{r} / \mathrm{min}$

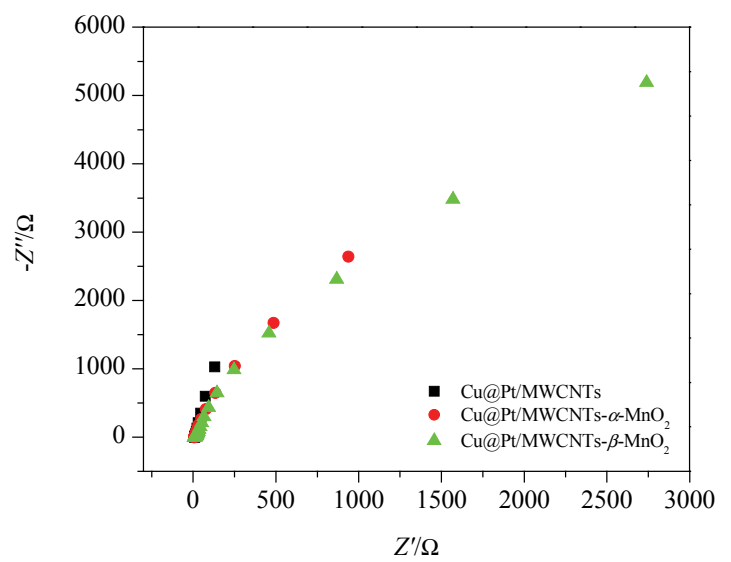

图 8 不同高铂载量电催化剂 $\mathrm{Cu} @ \mathrm{Pt} / \mathrm{MWCNTs}, \mathrm{Cu} @ \mathrm{Pt} / \mathrm{MWCNTs}-$ $\alpha-\mathrm{MnO}_{2}, \mathrm{Cu} @ \mathrm{Pt} / \mathrm{MWCNTs}-\beta-\mathrm{MnO}_{2}$ 的交流阻抗谱图

Figure 8 AC impedance spectra of different high Pt loading electrocatalysts $\quad \mathrm{Cu} @ \mathrm{Pt} / \mathrm{MWCNTs}, \quad \mathrm{Cu} @ \mathrm{Pt} / \mathrm{MWCNTs}-\alpha-\mathrm{MnO}_{2}, \quad \mathrm{Cu} @ \mathrm{Pt} /$ MWCNTs- $\beta-\mathrm{MnO}_{2}$.

Electrolyte $0.5 \mathrm{~mol} \cdot \mathrm{L}^{-1} \mathrm{H}_{2} \mathrm{SO}_{4}$. Sweep rate: $2 \mathrm{mV} \cdot \mathrm{s}^{-1}$. Frequency range: 0.1 $\mathrm{Hz} \sim 100 \mathrm{kHz}$, disturbance: $5 \mathrm{mV}$ 
高频区形成的曲线具有最小的斜率, 说明 $\mathrm{Cu} @ \mathrm{Pt} /$ MWCNTs- $\beta-\mathrm{MnO}_{2}$ 电催化剂在电荷传递过程中具有最 小的电阻, 有利于氧还原催化反应的进行.

$\mathrm{MnO}_{2}$ 能促进 $\mathrm{O}_{2}$ 还原的催化反应的可能机理是, 电 解液中的 $\mathrm{H}^{+}$与 $\mathrm{MnO}_{2}$ 中的 $\mathrm{O}^{2-}$ 结合, 形成 $\mathrm{OH}^{-}$, 从而形 成 $\mathrm{MnOOH}^{[14,24]}, \mathrm{MnOOH}$ 中 $\mathrm{Mn}^{3+}$ 对氧气的还原作用, 使 $\mathrm{MnO}_{2}$ 能够有效地促进氧还原反应(ORR)过程. 本文 选取了催化剂 $\mathrm{Cu} @$ Pt/MWCNTs 和 $\mathrm{Cu@Pt/MWCNTs-}$ $\beta-\mathrm{MnO}_{2}$ 进行了旋转环盘( RRDE)测试探究氧还原的路 径, 结果见图 9, 其中 $I_{\mathrm{R}}$ 为环电流, $I_{\mathrm{D}}$ 为盘电流. 从环电 流图可以看出, 催化剂 $\mathrm{Cu} @$ Pt/MWCNTs 和 $\mathrm{Cu} @$ Pt/ MWCNTs- $\beta-\mathrm{MnO}_{2}$ 的环电流密度均很小, 且后者比前者 更小, 说明二者在环上产生的 $\mathrm{H}_{2} \mathrm{O}_{2}$ 量都很小. 盘电流图 为不同转速下 $\mathrm{Cu} @ \mathrm{Pt} / \mathrm{MWCNTs}$ 催化剂氧还原的 LSV 曲线. 由该图可以看出, 随着转速增大, 电流逐渐增大, 氧的还原能力逐渐增强. 根据 Koutecky-Levich 方 程 ${ }^{[25,26]}$ :

$$
J^{-1}=J_{\mathrm{k}}^{-1}+\left(0.62 n F D^{2 / 3} c \mu^{-1 / 6} w^{1 / 2}\right)^{-1}
$$

式中 $D$ 为扩散系数, $D=1.9 \times 10^{-5} \mathrm{~cm}^{2} / \mathrm{s}^{2}, \mathrm{O}_{2}$ 在溶液中的 溶解度 $c=1.18 \times 10^{-6} \mathrm{~mol} / \mathrm{cm}^{3}, \mathrm{O}_{2}$ 在溶液中的动力学粘 度 $\mu=8.93 \times 10^{-3} \mathrm{~cm}^{2} / \mathrm{s}$, 法拉第常数 $F=96485$, 选取 $0.2 \mathrm{~V}$ 下不同转速的盘电流值作图, 见图 9a 内插图. 由 内插图的斜率即 $\left(0.62 n F D^{2 / 3} C \mu^{-1 / 6}\right)^{-1}$ 计算得到 $n$ 近似 为 4, 即 $\mathrm{Cu} @ \mathrm{Pt} / \mathrm{MWCNTs}$ 催化剂的氧还原过程为 $4 \mathrm{e}$ 的 还原路径, 对比环电流图所得到的 $\mathrm{Cu} @ \mathrm{Pt} / \mathrm{MWCNTs}-$ $\mathrm{MnO}_{2}$ 的测试结果, 可以说明, $\mathrm{Cu} @ \mathrm{Pt} / \mathrm{MWCNTs}-\mathrm{MnO}_{2}$ 催化剂也是采取的 $4 \mathrm{e}$ 还原路径, 与文献[19]相吻合. 说 明 $\mathrm{MnO}_{2}$ 的加入可以加速 $\mathrm{H}_{2} \mathrm{O}_{2}$ 的分解, 使得氧化还原过 程进行得更彻底.

\section{3 结论}

本文使用两步化学还原法以及水热法分别制备了 $\mathrm{Cu} @ \mathrm{Pt} / \mathrm{MWCNTs}$ 以及 $\alpha-\mathrm{MnO}_{2}$ 和 $\beta-\mathrm{MnO}_{2}$, 通过超声法 分别将其进行掺杂形成 $\mathrm{Cu} @ \mathrm{Pt} / \mathrm{MWCNTs}-\alpha-\mathrm{MnO}_{2}$ 和 $\mathrm{Cu} @ \mathrm{Pt} / \mathrm{MWCNTs}-\beta-\mathrm{MnO}_{2}$ 电催化剂, 经过一系列形貌 表征和性能测试, 得到如下结论:

(1) 利用 XRD, HRTEM 等方法表征了 $\mathrm{Cu} @ \mathrm{Pt}$ 纳米 颗粒的核壳结构, 其中 $\mathrm{Cu} @ \mathrm{Pt}$ 纳米颗粒的大小为 $6 \sim 8$ $\mathrm{nm} ; \alpha-\mathrm{MnO}_{2}$ 晶体呈线性, 直径约为 $20 \sim 40 \mathrm{~nm}$, 长度约 为 $2 \sim 4 \mu \mathrm{m}, \beta-\mathrm{MnO}_{2}$ 晶体呈六棱柱的形状, 直径约为 1.5 $\mu \mathrm{m}$, 长度约为 $4 \sim 6 \mu \mathrm{m}$.

(2) $\mathrm{Cu} @ \mathrm{Pt} / \mathrm{MWCNTs}-\mathrm{MnO}_{2}$ 电催化剂的催化性能测 试表明, 掺杂有 $\beta-\mathrm{MnO}_{2}$ 的 $\mathrm{Cu} @ \mathrm{Pt} / \mathrm{MWCNTs}$ 电催化剂 具有较高的氧还原催化效率, 其电化学活性面积最大为 $71.1 \mathrm{~m}^{2} \cdot \mathrm{g}^{-1}$.

(3) 通过计时电流和交流阻抗等电化学性能测试, 表明 $\mathrm{Cu} @ \mathrm{Pt} / \mathrm{MWCNTs}-\beta-\mathrm{MnO}_{2}$ 电催化剂与 $\mathrm{Pt} / \mathrm{MWCNTs}$ 相比具有更高的稳态电流和更小的电荷转移电阻, 且
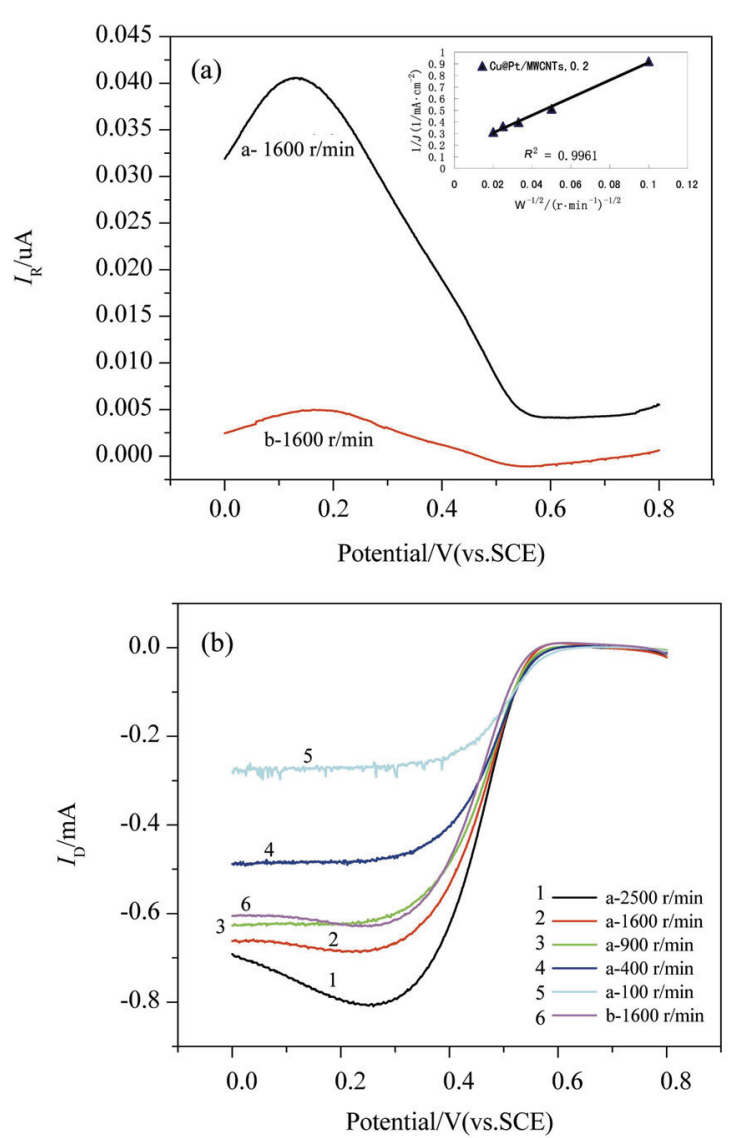

图 9 不同电催化剂的旋转环盘测试曲线

Figure 9 RRDE curves of different electrocatalysts

(a) $\mathrm{Cu} @ \mathrm{Pt} / \mathrm{MWCNTs}$; (b) $\mathrm{Cu} @ \mathrm{Pt} / \mathrm{MWCNTs}-\beta-\mathrm{MnO}_{2}$. Electrolyte: $0.5 \mathrm{~mol} \cdot$ $\mathrm{L}^{-1} \mathrm{H}_{2} \mathrm{SO}_{4}$. Sweep rate: $2 \mathrm{mV} \cdot \mathrm{s}^{-1}$. Rotation rate: $2500 \sim 100 \mathrm{r} / \mathrm{min}$

$\mathrm{Cu} @ \mathrm{Pt} / \mathrm{MWCNTs}-\mathrm{MnO}_{2}$ 催化剂在催化氧还原过程中, 采取的是 $4 \mathrm{e}$ 还原路径.

\section{4 实验部分}

\subsection{Cu@Pt/MWCNTs 电催化剂的制备}

将适量的 $\mathrm{CuSO}_{4} \cdot 5 \mathrm{H}_{2} \mathrm{O}$ 、柠檬酸和多壁碳纳米管固 体粉末分散在乙二醇溶液中, 超声分散 $2 \mathrm{~h}$, 油浴加热 至 $140{ }^{\circ} \mathrm{C}$, 使用 $\mathrm{KOH} / \mathrm{EG}$ 碱液调节并保持反应体系的 $\mathrm{pH}$ 至 10 , 回流 $4 \mathrm{~h}$, 冷却至室温, 洗涤、过滤、烘干、 研磨后得到 $\mathrm{Cu} / \mathrm{MWCNTs}$ 黑色固体粉末. 将适量的 $\mathrm{Cu} / \mathrm{MWCNTs}$ 黑色固体粉末分散在乙二醇溶液中, 超声 分散 $2 \mathrm{~h}$, 加入适量 $40 \mathrm{mg} \cdot \mathrm{mL}^{-1}$ 的 $\mathrm{H}_{2} \mathrm{PtCl}_{6} \cdot 6 \mathrm{H}_{2} \mathrm{O}$ 溶液, 油浴加热至 $90{ }^{\circ} \mathrm{C}$, 使用 $\mathrm{KOH} / \mathrm{EG}$ 碱液调节并保持反应 体系的 $\mathrm{pH}$ 至 7 8, 回流 $4 \mathrm{~h}$, 冷却至室温, 洗涤、过滤、 烘干、研磨后得到 $\mathrm{Cu} @ \mathrm{Pt} / \mathrm{MWCNTs}$ 电催化剂黑色固体 粉末.

\section{$4.2 \mathrm{Cu} @ \mathrm{Pt} / \mathrm{MWCNTs}-\mathrm{MnO}_{2}$ 电催化剂的制备}

称取适量的 $\mathrm{KMnO}_{4}$ 固体粉末和 $\mathrm{Mn}\left(\mathrm{NO}_{3}\right)_{2}$ 溶液分别 超声溶解于去离子水中, 将上述两种溶液混合, 摚拌均 匀成稳定的悬浊液, 转移至聚四氟乙烯为祄里的高压反 
应釜内, 程序升温至 $160{ }^{\circ} \mathrm{C}$, 反应 $8 \mathrm{~h}$ 后取出, 经冷却、 洗涤、过滤、干燥, 研磨制得棕色粉末 $\alpha-\mathrm{MnO}_{2}$. 将上述 反应延长至 $72 \mathrm{~h}$ 后经同样方法处理得到灰黑色粉末 $\beta-\mathrm{MnO}_{2}$.

通过超声法将 $\alpha-\mathrm{MnO}_{2}$ 和 $\beta-\mathrm{MnO}_{2}$ 与 $\mathrm{Cu} @ \mathrm{Pt} /$ MWCNTs 在乙醇溶液中进行掺杂, 形成 $\mathrm{Cu} @ \mathrm{Pt}$ / MWCNTs- $\mathrm{MnO}_{2}$ 电催化剂, 该电催化剂中各活性组分 的含量如表 2 .

表 $2 \mathrm{Cu} @ \mathrm{Pt} / \mathrm{MWCNTs}-\mathrm{MnO}_{2}$ 电催化剂中各活性组分的含量

Table 2 Active component content of $\mathrm{Cu} @ \mathrm{Pt} / \mathrm{MWCNTs}-\mathrm{MnO}_{2}$ electrocatalysts

\begin{tabular}{lcccc}
\hline 电催化剂 & $\mathrm{Cu} / \mathrm{mg}$ & $\mathrm{Pt} / \mathrm{mg}$ & $\alpha-\mathrm{MnO}_{2} / \mathrm{mg}$ & $\beta-\mathrm{MnO}_{2} / \mathrm{mg}$ \\
\hline $\mathrm{Cu} @$ Pt/MWCNTs- $\alpha-\mathrm{MnO}_{2}$ & 5 & 35 & 10 & - \\
$\mathrm{Cu} @ \mathrm{Pt} / \mathrm{MWCNTs}-\beta-\mathrm{MnO}_{2}$ & 5 & 35 & - & 10 \\
\hline
\end{tabular}

\section{References}

[1] Kumar, G. S.; Raja, M.; Parthasarathy, S. Electrochim. Acta 1995, 40, 285

[2] Pan, Y.; Zhang, F.; Wu, K.; Lu, Z. Y.; Chen, Y.; Zhou, Y. M.; Tang, Y. W.; Lu, T. H. Int. J. Hydrogen Energy 2012, 37, 2993.

[3] Noto, V. D.; Negro, E. J. Power Sources 2010, 195, 638.

[4] Kadirgan, F.; Kannan, A. M.; Atilan, T.; Beyhan, S.; Ozenler, S. S.; Suzer, S.; Yörür, A. Int. J. Hydrogen Energy 2009, 34, 9450.

[5] Tasic, G. S.; Miljanic, S. S.; Kaninski, M. P. M.; Saponjic, D. P.; Nikolica, V. M. Electrochem. Commun. 2009, 11, 2097.

[6] Wang, W.; Wang, R. F.; Ji, S.; Feng, H. Q.; Wang, H.; Lei, Z. Q. J. Power Sources 2010, 195, 3498.

[7] Mania, P.; Srivastava, R.; Strasserc, P. J. Power Sources 2011, 196, 666.
[8] Wang, H.; Wang, R. F.; Li, H.; Wang, Q. F.; Kang, J.; Lei, Z. Q. Int. J. Hydrogen Energy 2011, 36, 839.

[9] Wang, R. F.; Wang, H.; Wei, B. X.; Wang, W.; Lei, Z. Q. Int. J. Hydrogen Energy 2010, 35, 10081.

[10] Yang, X. J.; Cheng, F. Y.; Tao, Z. L.; Chen, J. J. Power Sources 2011, 196, 2785.

[11] Wang, G. X.; Wu, H. M.; Wexler, D.; Liu, H. K.; Savadogo, Q. J. Power Sources 2010, 195, 289.

[12] Zhu, H.; Li, X. W.; Wang, F. H. Int. J. Hydrogen Energy 2011, 36, 9151.

[13] Cao, Y. L.; Yang, H. X.; Ai, X. P.; Xiao, L. F. J. Electroanal. Chem. 2003, 557, 127.

[14] Wei, Z. D.; Ji, M. B.; Hong, Y.; Sun, C. X.; Chan, S. H.; Shen, P. K. J. Power Sources 2006, 160, 246.

[15] Kordesch, K. Battery Packs (I): Manganese Dioxide, China Light Industry Press, Beijing, 1981, p. 313.

[16] Lu, M.; Kharkwal, S.; Ng, H. Y.; Li, S. F. Y. Biosens. Bioelectron. 2011, 26, 4728 .

[17] Zhou, C. M.; Wang, H. J.; Peng, F.; Liang, J. H.; Yu, H.; Yang, J. Langmuir 2009, 25, 7711 .

[18] Yu, X. H.; He, J. H.; Wang, D. H.; Hu, Y. C.; Tian, H.; He, Z. C. J. Phys. Chem. C 2012, 116, 851.

[19] Trogadas, P.; Ramani, V. J. Power Sources 2007, 174, 159.

[20] Sarkar, A.; Manthiram, A. J. Phys. Chem. C 2010, 114, 4725.

[21] Luo, J.; Maye, M. M.; Petkov, V.; Kariuki, N. N.; Wang, L. Y.; Njoki, P.; Mott, D.; Lin, Y.; Zhong, C. J. Chem. Mater. 2005, 17, 3086 .

[22] Wang, R. F.; Li, H.; Feng, H. Q.; Wang, H.; Lei, Z. Q. J. Power Sources 2010, 195, 1099.

[23] Ban, M.; Qi, D. D.; Xia, D. G. Funct. Mater. 2004, 35, 1861. (班旻, 戚道铎, 夏定国, 功能材料, 2004, 35, 1861.)

[24] Wang, J.-G.; Yang, Y.; Huang, Z.-H.; Kang, F. Y. Mater. Chem. Phys. 2012, 133, 437.

[25] Paulus, U. A.; Wokaun, A.; Scherer, G. G. J. Phys. Chem. B 2002, $106,4181$.

[26] Shao, M. H.; Huang, T.; Liu, P.; Zhang, J.; Sasaki, K.; Vukmirovic, M. B.; Adzic, R. R. Langmuir 2006, 22, 10409. 